This item was submitted to Loughborough's Research Repository by the author.

Items in Figshare are protected by copyright, with all rights reserved, unless otherwise indicated.

\title{
Solvent mediated interactions close to fluid-fluid phase separation: microscopic treatment of bridging in a soft-core fluid
}

PLEASE CITE THE PUBLISHED VERSION

http://dx.doi.org/10.1063/1.1855878

PUBLISHER

(c) American Institute of Physics

VERSION

VoR (Version of Record)

LICENCE

CC BY-NC-ND 4.0

\section{REPOSITORY RECORD}

Archer, Andrew J., Robert Evans, Roland Roth, and M. Oettel. 2019. "Solvent Mediated Interactions Close to Fluid-fluid Phase Separation: Microscopic Treatment of Bridging in a Soft-core Fluid". figshare. https://hdl.handle.net/2134/16052. 


\section{A The Journal of Chemical Physics}

\section{Solvent mediated interactions close to fluid-fluid phase separation: Microscopic treatment of bridging in a soft-core fluid}

\section{A. J. Archer, R. Evans, R. Roth, and M. Oettel}

Citation: The Journal of Chemical Physics 122, 084513 (2005); doi: 10.1063/1.1855878

View online: http://dx.doi.org/10.1063/1.1855878

View Table of Contents: http://scitation.aip.org/content/aip/journal/jcp/122/8?ver=pdfcov

Published by the AIP Publishing

\section{Articles you may be interested in}

Solvent annealing induced phase separation and dewetting in PMMA/SAN blend film: Film thickness and solvent dependence

J. Chem. Phys. 138, 244907 (2013); 10.1063/1.4811471

Solvent mediated interactions between model colloids and interfaces: A microscopic approach

J. Chem. Phys. 131, 124704 (2009); 10.1063/1.3212888

Phase separation of two-dimensional Coulombic crystals of mesoscale dipolar particles from mesoscale polarizable "solvent"

Appl. Phys. Lett. 94, 044102 (2009); 10.1063/1.3052616

Solvent-mediated interactions and solvation close to fluid-fluid phase separation:A density functional treatment J. Chem. Phys. 118, 9726 (2003); 10.1063/1.1570406

Polymer-polymer interaction parameters for homopolymers and copolymers from light scattering and phase separation experiments in a common solvent

J. Chem. Phys. 115, 9536 (2001); 10.1063/1.1412867

\section{AIP $\mid$ chaos \\ CALL FOR APPLICANTS Seeking new Editor-in-Chief}




\title{
Solvent mediated interactions close to fluid-fluid phase separation: Microscopic treatment of bridging in a soft-core fluid
}

\author{
A. J. Archer ${ }^{\mathrm{a})}$ and R. Evans \\ H. H. Wills Physics Laboratory, University of Bristol, Bristol BS8 1TL, United Kingdom \\ R. Roth and M. Oettel \\ Max-Planck-Institut für Metallforschung, Heisenbergstrasse 3, 70569 Stuttgart, Germany and ITAP, \\ Universität Stuttgart, Pfaffenwaldring 57, 70569 Stuttgart, Germany
}

(Received 22 November 2004; accepted 10 December 2004; published online 22 February 2005)

\begin{abstract}
Using density functional theory we calculate the density profiles of a binary solvent adsorbed around a pair of big solute particles. All species interact via repulsive Gaussian potentials. The solvent exhibits fluid-fluid phase separation, and for thermodynamic states near to coexistence the big particles can be surrounded by a thick adsorbed "wetting" film of the coexisting solvent phase. On reducing the separation between the two big particles we find there can be a "bridging" transition as the wetting films join to form a fluid bridge. The effective (solvent mediated) potential between the two big particles becomes long ranged and strongly attractive in the bridged configuration. Within our mean-field treatment the bridging transition results in a discontinuity in the solvent mediated force. We demonstrate that accounting for the phenomenon of bridging requires the presence of a nonzero bridge function in the correlations between the solute particles when our model fluid is described within a full mixture theory based upon the Ornstein-Zernike equations.
\end{abstract}

(C) 2005 American Institute of Physics. [DOI: 10.1063/1.1855878]

\section{INTRODUCTION}

Big solute particles (e.g., colloids) immersed in a solvent of smaller particles interact with each other by an effective potential which is the sum of their direct interaction and a solvent mediated (SM) potential. Even when the direct interaction consists solely of two-body terms, the SM potential usually contains higher-body contributions of all orders which are determined formally by integrating out the solvent degrees of freedom. This conceptual framework yields, in principle, a much simpler effective Hamiltonian which involves only the coordinates of the big particles. ${ }^{1}$ In certain systems the two-body term in the SM potential may dominate completely the corresponding direct interaction. A wellknown example is a suspension of big hard-sphere colloids in a solvent of small hard spheres. There the SM potential between the colloids is termed the depletion interaction, and this is the only contribution to the effective potential for separations greater than the big hard-sphere diameter. ${ }^{2}$ In the case of a (nonhard) solvent which is at a state point near to fluid-fluid phase separation, big solute particles can be surrounded by a thick adsorbed "wetting" film of the coexisting solvent phase. ${ }^{3}$ If two such big particles become sufficiently close, there can be a "bridging transition" as the wetting films surrounding the two big particles join to form a fluid bridge of the wetting phase-see, for example, Ref. 4 and references therein. In wet granular media these bridging (or capillary) forces lead to strong and very short-ranged interactions. Tip-substrate interactions in atomic force microscopy can be long ranged due to the formation of capillary

${ }^{a)}$ Electronic mail: Andrew.Archer@bristol.ac.uk bridges. ${ }^{5}$ Long-ranged attractive interactions are also surmised for hydrophobic molecules in water at ambient conditions. ${ }^{6}$ Bridging is also a purported mechanism for driving colloidal flocculation. ${ }^{7}$

In previous work, ${ }^{8,9}$ the wetting of a binary solvent around a single big particle and the influence of these thick adsorbed films on the effective SM potential between two big particles was investigated for a particular model fluid, namely, the generalization to mixtures of the Gaussian core model (GCM) ${ }^{1,8-18}$ A Gaussian potential provides a good approximation for the effective potential between the centers of mass of polymers in solution. ${ }^{1,19,20}$ The approach to calculating the SM potentials was based upon the theory developed by Roth $e t a l .{ }^{2}$ - henceforth referred to as the "insertion method." The insertion method works within the framework of density functional theory (DFT) (Ref. 21) and uses as input the density profiles calculated around a single big particle in order to calculate the SM potential between a pair of big particles. ${ }^{2,8,9}$ Although the insertion method is formally exact, in practice, one must employ an approximation for the free energy functional of the mixture of big and small particles. ${ }^{2}$ For state points near to coexistence we found thick adsorbed films around the big particles resulting in long ranged, strongly attractive SM potentials whose range was determined by the thickness of the wetting film. However, using the insertion method, we were unable to detect any direct sign of bridging in the SM potential. ${ }^{8,9}$

The present work can be viewed as going a significant step further than Refs. 8 and 9. Here we investigate the same system: two large solute Gaussian particles, immersed in a binary GCM solvent near to phase separation. However, whereas the previous work used the elegant insertion 
method, the present work can be viewed as the "brute-force" approach to the problem. Using an accurate DFT for the binary GCM solvent of small particles ${ }^{1,8,9,16,17}$ we calculate explicitly the solvent density profiles around a fixed pair of the big GCM particles, treating the latter as external potentials, and determine the resulting grand potential. By repeating this calculation for a range of values of the separation between the centers of the two big particles we obtain the SM potential. We find, within the present (mean-field) DFT approach, that when thick adsorbed films are present there can be a bridging transition as the separation between the two big particles is decreased, i.e., the formation of a bridging configuration gives rise to a discontinuity in the derivative of the SM potential. Bridging has been investigated previously within (coarse-grained) local DFT (in contrast to our nonlocal treatment) in the recent study of Stark et al. ${ }^{22}$ for big hard spherical colloids immersed in an isotropic liquid crystal host close to the isotropic-nematic phase boundary. Similarly, Andrienko et al. ${ }^{23}$ calculated bridging density profiles of a solvent adsorbed between a big colloid and a planar wall using a local DFT.

We also investigate the SM potential between two big GCM particles in a region of the solvent phase diagram near the binodal but lying outside the single particle thin-thick adsorbed film transition line, ${ }^{9}$ where a single big particle does not have a thick adsorbed wetting film of the coexisting solvent phase around it. Adsorption still influences strongly the SM potential. We find an analog of capillary condensation; as the two big particles become sufficiently close, the composite object is large enough to induce condensation of the coexisting solvent phase around the pair of big particles. This effect is somewhat different from that which can occur between two big hard-core particles in a solvent near to coexistence. When a pair of such particles are sufficiently close, a bridge of the coexisting phase can condense in the gap between the two big particles, without there being thick wetting films adsorbed on each of the big particles. ${ }^{4}$ In the present soft-core system the strong adsorption is not confined to the space between the big particles, rather it extends through the whole region in which the two big particles are situated. This local condensation also results in a jump in the SM force between the two big particles with the SM potential becoming strongly attractive for small separations.

In the final part of the present work we relate our results for the SM potential to an approach for calculating the SM potential based upon the mixture Ornstein-Zernike (OZ) equations. ${ }^{24-26}$ By solving the $\mathrm{OZ}$ equations together with a closure relation one can calculate the various fluid correlation functions. It is well known that if one makes a diagrammatic expansion for the fluid correlation functions the hypernetted chain (HNC) closure approximation neglects a certain class of (bridge) diagrams which, taken together, is termed the bridge function. ${ }^{24}$ We show that in order to account for the phenomenon of bridging of solvent between big particles within a $\mathrm{OZ}$ approach to the fluid structure, one must incorporate an accurate theory for the bridge diagrams.

The paper is laid out as follows. In Sec. II we describe briefly our model fluid, the GCM, and the DFT used to calculate the solvent density profiles and the SM potential be- tween two big solute GCM particles. Section III presents results for the density profiles and SM potentials in the regime where there are thick adsorbed films around a single big particle, resulting in a bridging transition when two big particles are sufficiently close together. In Sec. IV we present a simple analytic "capillarity" approximation which describes qualitatively the onset of the bridging transitions that we find. Section $\mathrm{V}$ describes the effect of the formation of a thick adsorbed film around a pair of big particles, in the portion of the phase diagram where there is no thick film around a single big particle and Sec. VI describes our demonstration that bridging between big particles is related to the bridge function. Finally, in Sec. VII, we discuss our results and draw some conclusions.

\section{MODEL FLUID AND SM POTENTIALS}

We determine the SM potential between two big $(B)$ Gaussian particles immersed in a binary solvent of smaller Gaussian particles. The GCM, in which the particles of species $i$ and $j$ interact via purely repulsive Gaussian potentials

$$
v_{i j}(r)=\epsilon_{i j} \exp \left(-r^{2} / R_{i j}^{2}\right),
$$

is a simple model for polymers in solution ${ }^{1,12,13,16,17,20}$ (in particular, Ref. 1 provides a good general introduction to the GCM). For the binary GCM solvent we choose pair potential parameters corresponding to a binary mixture of polymers of length ratio 2:1, as were used in previous work on this model fluid. ${ }^{8,9,16,17}$ The values are $R_{22} / R_{11}=0.665, R_{12} / R_{11}=0.849$, $\beta \epsilon_{11}=\beta \epsilon_{22}=2.0\left(\beta=1 / k_{B} T\right)$, and $\epsilon_{12} / \epsilon_{11}=0.944 . R_{11}$ is the basic length scale in the system. For this choice of parameters the binary mixture exhibits fluid-fluid phase separation. The phase diagram of this binary solvent is plotted in the total density $\rho^{0}=\rho_{1}^{0}+\rho_{2}^{0}$ versus concentration $x=\rho_{2}^{0} / \rho^{0}$ plane in Fig. $1\left(\rho_{\nu}^{0}\right.$ are the bulk densities of the small particles of species $\nu=1,2)$ - see also Ref. 16.

The SM potential between two big particles, labeled $A$ and $B$, with centers at $\mathbf{r}_{A}$ and $\mathbf{r}_{B}$, separated by a distance $h$, is given by the difference in the grand potential,

$$
W_{A B}(h)=\Omega\left(\left|\mathbf{r}_{A}-\mathbf{r}_{B}\right|=h\right)-\Omega\left(\left|\mathbf{r}_{A}-\mathbf{r}_{B}\right|=\infty\right) .
$$

This result can be reexpressed (trivially) in terms of excess grand potentials, $\omega_{e x}^{i} \equiv \Omega-\Omega_{b}$, where $i=A, B$, and $\Omega_{b}$ is the grand potential of the bulk solvent in the situation where there are no big particles present. Then,

$$
W_{A B}(h)=\omega_{e x}^{A B}\left(\left|\mathbf{r}_{A}-\mathbf{r}_{B}\right|=h\right)-\omega_{e x}^{A}-\omega_{e x}^{B} .
$$

Note that $\omega_{e x}^{i}$, the excess grand potential for inserting a single big particle of species $i$, is equal to $\mu_{e x}^{i}$, the excess chemical potential of big species $i$ in the limit of the bulk density of this species $\rho_{i}^{0} \rightarrow 00^{2,8,9}$ The effective pair potential between two identical big particles is then the sum of the bare interaction $v_{B B}(r)$ and the SM potential,

$$
v_{B B}^{e f f}(h)=v_{B B}(h)+W_{B B}(h) .
$$




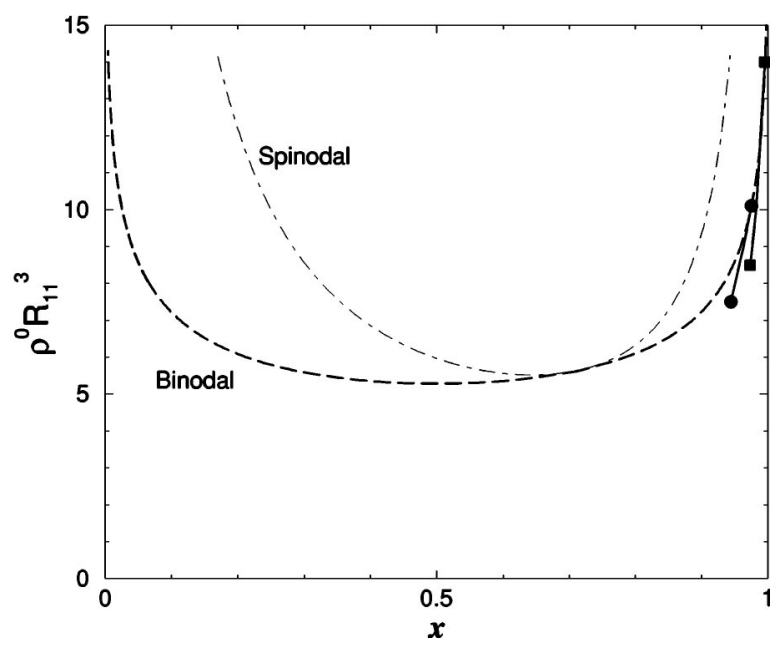

FIG. 1. The bulk phase diagram for a binary mixture of GCM particles with $\epsilon_{12} / \epsilon_{11}=0.944$ and $R_{22} / R_{11}=0.665$, equivalent to a mixture of two polymers with length ratio $2: 1$ (see also Ref. 16). $\rho^{0}$ is the total density and $x$ is the concentration of the smaller species 2 . The solid line whose ends are denoted by filled circles is the thin-thick adsorbed film transition of the binary fluid adsorbed around a single big GCM particle with pair potential parameters $\beta \epsilon_{B 1}=1.0, \beta \epsilon_{B 2}=0.8, R_{B 1} / R_{11}=5.0$, and $R_{B 2} / R_{11}=4.972$-see Ref. 9. It meets the binodal at the wetting point (upper circle) with $x=0.975$ and $\rho^{0} R_{11}^{3}=10.1$ [note these values differ slightly from the result quoted in Ref. 9-see footnote (Ref. 30)] and terminates at a critical point (lower circle) with $x=0.94$ and $\rho^{0} R_{11}^{3}=7.5$. The solid line whose ends are denoted by filled squares is the thin-thick adsorbed film transition of the binary fluid adsorbed around a composite pair of the same big GCM particles at zero separation $h=0$. This transition line meets the binodal (upper square) at $x=0.995$ and $\rho^{0} R_{11}^{3}=14$ and terminates at a critical point (lower square) with $x=0.973$ and $\rho^{0} R_{11}^{3}=8.5$.

$$
v_{B B}^{e f f}(h)=-k_{B} T \ln g_{B B}(h),
$$

where $g_{B B}$ is the big-big radial distribution function in the limit of the big particle bulk density $\rho_{B}^{0} \rightarrow 0$. In the present work we use DFT to obtain the quantities $\omega_{e x}^{A B}\left(\left|\mathbf{r}_{A}-\mathbf{r}_{B}\right|=h\right)$ and $\omega_{e x}^{i}$

In DFT one calculates the solvent one-body density profiles, $\left\{\rho_{\nu}(\mathbf{r})\right\}$, for a given set of external potentials, $\left\{V_{\nu}(\mathbf{r})\right\}$, by minimizing the grand potential functional, ${ }^{21}$

$$
\begin{aligned}
\Omega_{V}\left[\left\{\rho_{\nu}\right\}\right]= & \mathcal{F}_{i d}\left[\left\{\rho_{\nu}\right\}\right]+\mathcal{F}_{e x}\left[\left\{\rho_{\nu}\right\}\right] \\
& -\sum_{\nu} \int \mathrm{d} \mathbf{r} \rho_{\nu}(\mathbf{r})\left[\mu_{\nu}-V_{\nu}(\mathbf{r})\right]
\end{aligned}
$$

where $\mu_{\nu}$ are the chemical potentials for the two species, $\nu$ $=1,2$, of solvent particles. The ideal gas part of the intrinsic Helmholtz free energy functional is

$$
\mathcal{F}_{i d}\left[\left\{\rho_{\nu}\right\}\right]=k_{B} T \sum_{\nu} \int \mathrm{d} \mathbf{r} \rho_{\nu}(\mathbf{r})\left[\ln \left(\Lambda_{\nu}^{3} \rho_{\nu}(\mathbf{r})\right)-1\right]
$$

where $\Lambda_{\nu}$ is the thermal de Broglie wavelength of species $\nu$, and $\mathcal{F}_{e x}\left[\left\{\rho_{\nu}\right\}\right]$ is the excess part of the intrinsic Helmholtz free energy functional. Minimizing Eq. (6) together with Eq. (7) one obtains the Euler-Lagrange equation

$$
0=k_{B} T \ln \Lambda_{\nu}^{3} \rho_{\nu}(\mathbf{r})-k_{B} T c_{\nu}^{(1)}(\mathbf{r})-\mu_{\nu}+V_{\nu}(\mathbf{r}),
$$

where

$$
c_{\nu}^{(1)}(\mathbf{r})=-\beta \frac{\delta \mathcal{F}_{e x}\left[\left\{\rho_{\nu}\right\}\right]}{\delta \rho_{\nu}(\mathbf{r})}
$$

is the one-body direct correlation function, which is a functional of $\left\{\rho_{\nu}\right\}$. In an exact treatment the density profiles $\left\{\rho_{\nu}\right\}$ satisfying Eq. (8) would yield the exact grand potential $\Omega$ as the minimum of $\Omega_{V \cdot}{ }^{21}$ At this point we also recall that the two-body direct correlation functions are given by the second functional derivative ${ }^{21}$

$$
c_{\nu, \xi}^{(2)}\left(\mathbf{r}, \mathbf{r}^{\prime}\right)=-\beta \frac{\delta^{2} \mathcal{F}_{e x}\left[\left\{\rho_{\nu}\right\}\right]}{\delta \rho_{\nu}(\mathbf{r}) \delta \rho_{\xi}\left(\mathbf{r}^{\prime}\right)} .
$$

For the GCM the following approximate excess Helmholtz free energy functional turns out, despite its simplicity, to be remarkably accurate at high densities $\rho^{0} R_{11}^{3} \gtrsim 5,{ }^{1,9,12,13,16,27}$

$$
\mathcal{F}_{e x}^{R P A}\left[\left\{\rho_{i}\right\}\right]=\frac{1}{2} \sum_{\nu, \xi} \int \mathrm{d} \mathbf{r} \int \mathrm{d} \mathbf{r}^{\prime} \rho_{\nu}(\mathbf{r}) \rho_{\xi}\left(\mathbf{r}^{\prime}\right) v_{\nu, \xi}\left(\left|\mathbf{r}-\mathbf{r}^{\prime}\right|\right),
$$

where $v_{\nu, \xi}(r)$ is the pair potential between the small solvent particles of species $\nu$ and $\xi$, given by Eq. (1). The functional, Eq. (11), is that which generates the random-phase approximation (RPA) closure, $c_{\nu, \xi}^{(2), R P A}\left(\mathbf{r}, \mathbf{r}^{\prime}\right)=-\beta v_{\nu, \xi}\left(\left|\mathbf{r}-\mathbf{r}^{\prime}\right|\right)$, for the pair direct correlation functions. ${ }^{\nu, 12,16,16}$ The higher the density, the more accurate is the RPA for this soft-core model. ${ }^{1}$

In the present work we choose the external potential to correspond to two fixed big Gaussian particles of the same size, separated by a distance $h$,

$$
\begin{aligned}
V_{\nu}(\mathbf{r})= & \epsilon_{B \nu} \exp \left(-(\mathbf{r}+\mathbf{h} / 2)^{2} / R_{B \nu}^{2}\right) \\
& +\epsilon_{B \nu} \exp \left(-(\mathbf{r}-\mathbf{h} / 2)^{2} / R_{B \nu}^{2}\right),
\end{aligned}
$$

with $\nu=1,2$ and where $\mathbf{h}$ is a vector along the $z$-axis, with $|\mathbf{h}|=h$, i.e., the centers of the big particles are at $z= \pm h / 2$. Throughout the present study we choose the external potential parameters to be $\beta \epsilon_{B 1}=1.0, \beta \epsilon_{B 2}=0.8, R_{B 1} / R_{11}=5.0$, and $R_{B 2} / R_{11}=4.972$, the same values as those used for the bigsmall particle pair potentials in much of the work in Refs. 8 and 9. With this external potential the solvent density profiles have cylindrical symmetry, i.e., the density profiles are functions $\rho_{\nu}(z, r)$, where the $z$-axis runs through the centers of the two big particles and $r$ is the radial distance from the $z$-axis. If the external potential on the solvent were exerted by hard particles, special care would be required to ensure that the hard boundary is compatible with the grid of the numerical calculations in order to avoid numerical artifacts in the contact density. ${ }^{28}$ One would have to employ either matching coordinate systems, such as the bispherical one used, e.g., in Refs. 22 and 29, or even more sophisticated finite-element methods with adaptive mesh size. ${ }^{23}$ One of the appealing features of the soft-core GCM used in the present investigation is that we can avoid this problem and perform our calculations on a uniform grid in cylindrical polar coordinates.

In Fig. 2 we display a typical density profile for a onecomponent solvent of particles of species 1, with the external potential given by Eq. (12) with $h / R_{11}=12$. Having calculated the solvent density profiles for a given separation $h$ of 


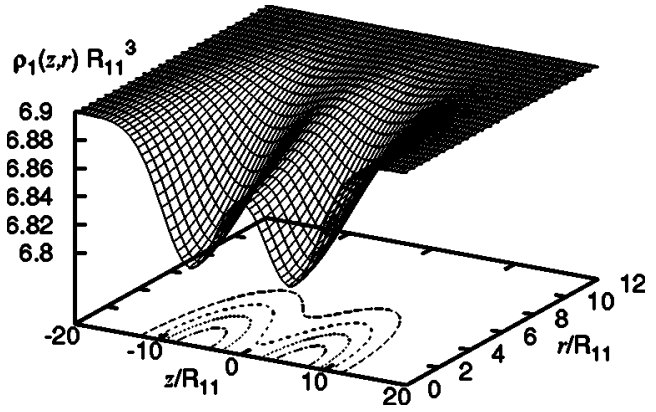

FIG. 2. The density profile of a one-component fluid of Gaussian particles, with bulk density $\rho_{1}^{0} R_{11}^{3}=6.9$, around a pair of big Gaussian particles, whose centers are located on the $z$ axis a distance $h / R_{11}=12$ apart. The contours in the $z-r$ plane correspond to $\rho_{1}(z, r)=6.82,6.84,6.86$, and 6.88 .

big particles, we can insert these into Eq. (6) to calculate the (excess) grand potential and the SM potential $W_{B B}(h)$ from Eq. (3). In Fig. 3 we display the SM potential between two big GCM particles, calculated for a one-component solvent with bulk density $\rho_{1}^{0} R_{11}^{3}=6.9$, i.e., the state point corresponding to the profiles in Fig. 2. Figure 3 should be compared with Fig. 2 of Ref. 9. The open circles are the results from the present "brute-force" calculation. The solid line is the result obtained using the insertion method, where one calculates only the solvent density profiles around an isolated, single big particle and then uses the general result, ${ }^{2}$

$$
\beta W_{B B}(h)=c_{B}^{(1)}\left(h \rightarrow \infty ; \rho_{B}^{0} \rightarrow 0\right)-c_{B}^{(1)}\left(h ; \rho_{B}^{0} \rightarrow 0\right),
$$

i.e., one calculates the difference in the excess chemical potential between inserting the second big particle a distance $h$ from the first and inserting it at $h=\infty$. As emphasized in the Introduction, Eq. (13) is formally exact when we know the exact free energy functional for a mixture of big and small particles. Here we use the same RPA functional (11) extended to include a third species $B$, in order to find an approximate $c_{B}^{(1)}$ in Eq. (13)—see Ref. 9 for more details. The results from the two different routes are almost indistinguish-

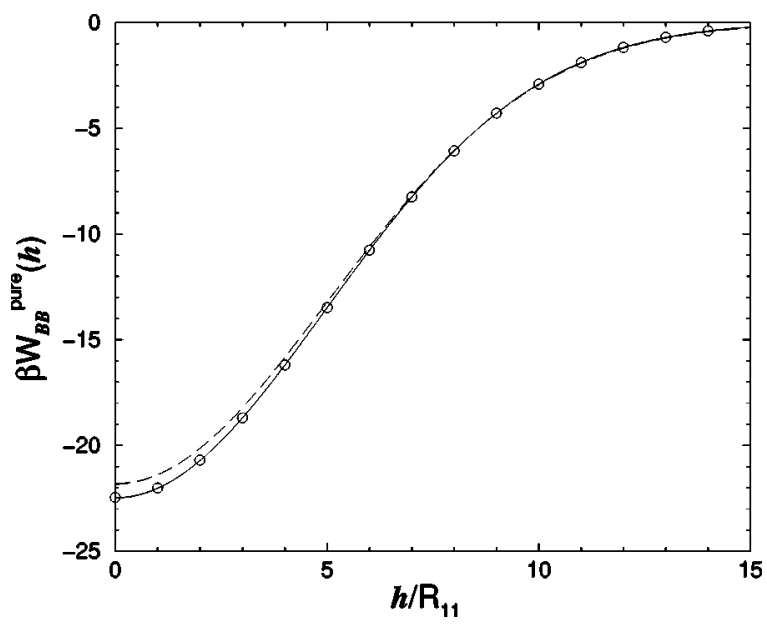

FIG. 3. The SM potential between two big GCM particles in a onecomponent solvent of small GCM particles, with bulk density $\rho_{1}^{0} R_{11}^{3}=6.9 . h$ is the separation between the two big particles. The solid line is the DFT insertion method results, the open circles are the results from the present brute-force calculation (the two are almost indistinguishable) and the dashed line is the analytic result, Eq. (14), obtained in Ref. 9.
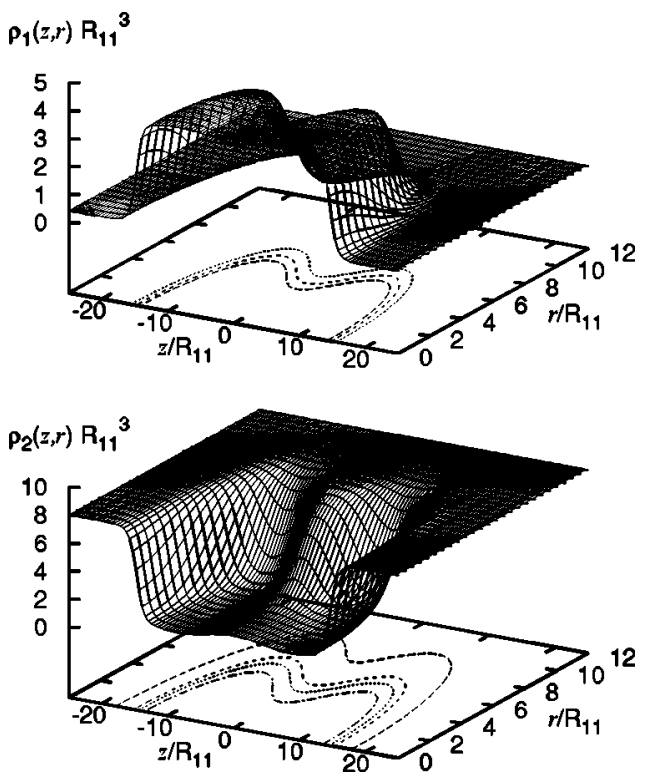

FIG. 4. Density profiles $\rho_{\nu}(z, r), \nu=1,2$, for a solvent with total density $\rho^{0} R_{11}^{3}=8.5$ and concentration $x=0.948$, a state near to phase separation located inside the single particle thin-thick adsorbed film transition line (see Fig. 1). The centers of the big particles are a distance $h / R_{11}=17$ apart. Note the presence of thick adsorbed (wetting) films and the fluid bridge between the particles. The contours, plotted in the $z-r$ plane, correspond to $\rho_{1}(z, r) R_{11}^{3}=1,2$, and 3 and $\rho_{2}(z, r) R_{11}^{3}=2-8$ in increments of 2 . The bridged configuration is the stable one for this value of $h / R_{11}$.

able for this point in the phase diagram, and generally for other state points where no thick adsorbed (wetting) films are present around the big particles. The dashed line in Fig. 3 corresponds to the analytic approximation for $W_{B B}(h)$ presented in Ref. 9,

$$
\beta W_{B B}^{\text {pure }}(h)=-(\pi / 2)^{3 / 2} \beta \epsilon_{B 1} \rho^{*} R_{B 1}^{3} \exp \left(-h^{2} / 2 R_{B 1}^{2}\right),
$$

where $\rho^{*}=\rho_{1}^{0} \beta \epsilon_{B 1} /\left(1+\pi^{3 / 2} \beta \epsilon_{11} R_{11}^{3} \rho_{1}^{0}\right)$. The agreement between this approximation and the result of the full numerical DFT calculations is remarkably good.

\section{THE SM POTENTIAL WHEN THERE ARE THICK ADSORBED FILMS: BRIDGING}

We now consider the case when thick adsorbed films develop around the big GCM particles. The circumstances in which this can occur are discussed in Refs. 8 and 9. In general there can be thick adsorbed films when the small solvent particles are in a state near to phase separation. For the present mixture, the big GCM particles favor species 1 of the small solvent particles, and so thick adsorbed films of the coexisting phase rich in species 1 can develop when the big particles are immersed in the solvent at a state point lying on the right hand side of the binodal, which is poor in species 1 . In Refs. 8 and 9 it was found that thick films develop via a thin-thick transition out of bulk coexistence. The locus of these transitions is shown as the solid line joining filled circles in Fig. 1. Note that this transition line meets the binodal at a wetting point whose density is somewhat higher than that quoted in Ref. 9. This discrepancy is associated with the existence of metastable minima in the free energy. ${ }^{30}$ In Figs. 4 and 5 we display density profiles calculated for a pair of big particles immersed in a binary solvent of small 

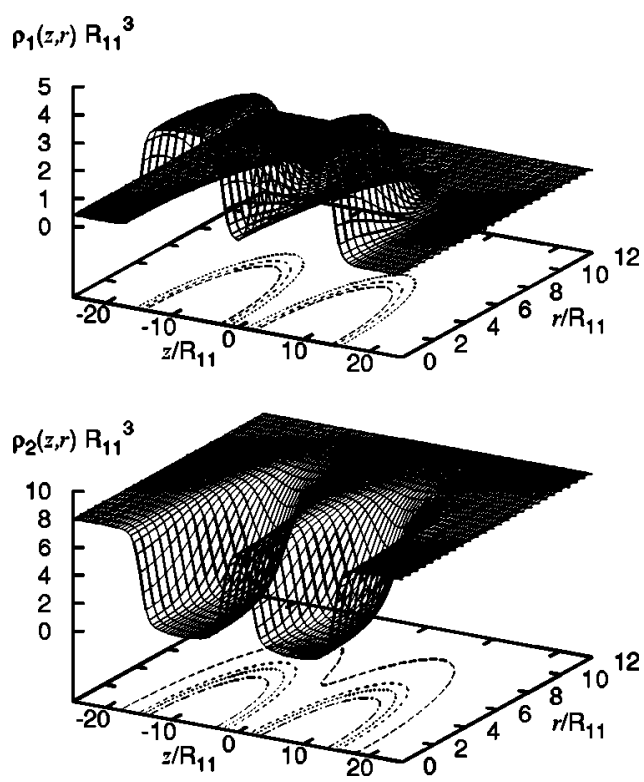

FIG. 5. Density profiles for the same state point and separation, $h / R_{11}=17$, as Fig. 4, but now there is no fluid bridge between the big particles. This configuration is metastable.

GCM particles with bulk density $\rho^{0} R_{11}^{3}=8.5$ and concentration $x=0.948$, a state point near to coexistence, located inside the single particle thin-thick adsorbed film transition line (see Fig. 1). (Figure 6 of Ref. 9 displays the solvent density profiles around a single big particle for this state point.) In Figs. 4 and 5 the centers of the big particles are a distance $h / R_{11}=17$ apart and there are thick adsorbed wetting films around the big particles. However, in Fig. 4 there is a fluid bridge between the two particles, whereas in Fig. 5 there is no fluid bridge. This second set of profiles corresponds to a metastable situation. For this state point the bridging transition occurs at a slightly larger separation $h_{t} / R_{11}=17.4$; this is where the bridged and unbridged configurations have equal grand potential. In Fig. 6 we display the SM potential $W_{B B}(h)$ for this state point. There are two distinct branches, corresponding to bridged and nonbridged configurations. For $h>h_{t}$ the unbridged configuration is the stable one, whereas for $h<h_{t}$ the bridged configuration becomes stable. Since the two branches of $W_{B B}(h)$ have different slopes there is a discontinuity in the SM force, $-\mathrm{d} W_{B B}(h) / \mathrm{d} h$, at $h_{t}$, the separation where the transition occurs. The extent of the metastable portions is substantial; these extend well beyond the equilibrium transition. This type of metastability, with accompanying hysteresis, was also found by Stark et $a l^{22}$ in their recent study of the bridging of the nematic wetting film between two colloids immersed in the isotropic phase of a liquid crystal. We display in Fig. 7 the SM potential calculated in the same way for a different point in the phase diagram, closer to the solvent bulk critical point, at a total density $\rho^{0} R_{11}^{3}=6.9$ and concentration $x=0.88$. This state point is also near to bulk coexistence (see Fig. 1). In both Figs. 6 and 7 we compare the SM potential calculated using the present bruteforce approach (solid lines) with the results obtained using the insertion method (dashed line) as described in Ref. 9. There is a significant difference between the results from the two methods; the insertion method does not capture the ex-

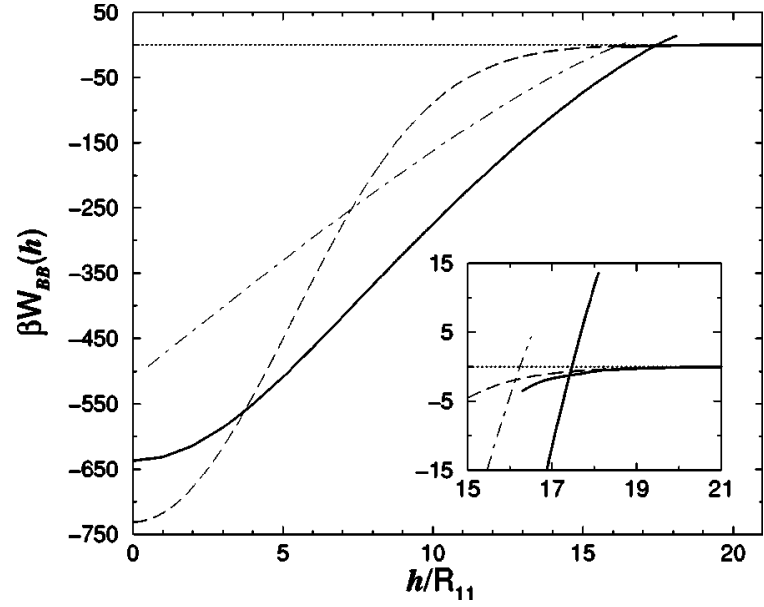

FIG. 6. The SM potential between two big GCM particles in a binary solvent of smaller particles for the same state point as in Figs. 4 and 5, i.e., with total bulk density $\rho^{0} R_{11}^{3}=8.5$ and concentration $x=0.948$. $h$ is the separation between the centers of the two big particles. The dashed line is the result for $W_{B B}(h)$ obtained using the insertion method, the dot-dashed line is the sharp-kink result (see text, Sec. IV) and the solid lines denote the results from the brute-force calculation. In the brute-force calculation, one finds that there are two branches for $W_{B B}(h)$ (see inset for more detail), each with a metastable portion. The branch with the smaller value of $W_{B B}(h)$ is stable. This corresponds to the configuration with no bridge for $h>h_{t}$, and to the bridged configuration for $h<h_{t}$. At $h_{t} / R_{11}=17.4$, where the two branches cross, there is a discontinuity in the gradient of $W_{B B}(h)$, i.e., there is a jump in the SM force at this separation.

istence of two distinct branches of the grand potential. Thus it does not appear to include explicitly the effects of a bridging transition. The insertion method does predict very strongly attractive SM potentials of a similar magnitude to those from full DFT, but does not yield the correct shape or range for $W_{B B}(h)$. In contrast we recall from Sec. II that in the regime where there are no thick adsorbed films, the results from the insertion method and the brute-force method are in good agreement.

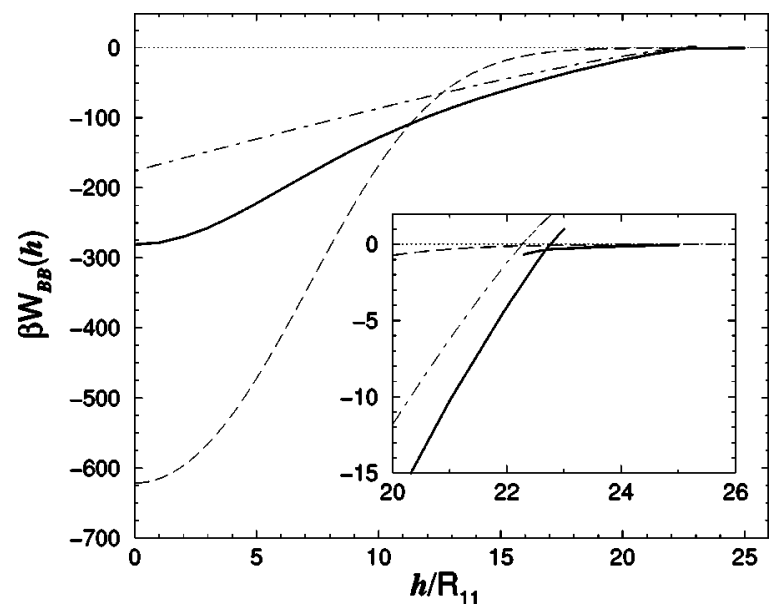

FIG. 7. The SM potential between two big GCM particles in a binary solvent of smaller particles near to phase separation, with total bulk density $\rho^{0} R_{11}^{3}=6.9$ and concentration $x=0.88$. $h$ is the separation between the two big particles. The dashed line is the result for the SM potential from the insertion method, the solid lines are the results from the brute-force calculation and the dot-dashed line is the sharp-kink result. In the inset we display a magnification of $W_{B B}(h)$ for large $h$, showing the two branches crossing at $h_{t} / R_{11}=22.7$ and giving rise to a jump in the SM force. 


\section{APPROXIMATION FOR THE SM POTENTIAL WHEN BRIDGING OCCURS}

In Ref. 9 we found that when there was a thick adsorbed film around a single big particle, a good approximation for the excess grand potential of a single big GCM particle immersed in a binary GCM solvent of small particles is

$$
\omega_{e x}^{B} \simeq \sum_{\nu=1}^{2} \pi^{3 / 2} \epsilon_{B \nu} R_{B \nu}^{3} \rho_{\nu}^{c o e x}+4 \pi l^{2} \gamma(l),
$$

where $\rho_{\nu}^{\text {coex }}$ are the solvent bulk densities in the coexisting phase, i.e., the phase that forms the adsorbed film. $l$ is the thickness of the adsorbed film $\left(l \sim R_{B \nu}\right.$, but we determine its value by calculating explicitly via DFT, the density profiles around a single big particle) and $\gamma(l)$ is the fluid-fluid surface tension, which we approximate by $\gamma(\infty)$, the surface tension of the planar free interface (this is calculated using the approach presented in Ref. 16). The first term in Eq. (15) is the excess grand potential for inserting a single big particle into the coexisting phase, obtained from the RPA bulk equation of state, ${ }^{9}$ and the second term is the contribution from forming a spherical fluid-fluid interface. Generalizing to two big particles we might therefore expect the following approximation to be reliable:

$$
\omega_{e x}^{B B}(h) \simeq 2 \sum_{\nu=1}^{2} \pi^{3 / 2} \epsilon_{B \nu} R_{B \nu}^{3} \rho_{\nu}^{\text {coex }}+A(l, h) \gamma^{\prime}(l, h),
$$

where $A(l, h)$ is the surface area of the fluid-fluid interface between the adsorbed film of the phase rich in species 1 which develops around the two big particles and the bulk fluid rich in species 2. $\gamma^{\prime}(l, h)$ is the surface tension, which we again approximate by $\gamma(\infty)$, the planar fluid-fluid interfacial tension. A similar sharp-kink or capillarity approach was used in Ref. 4 to investigate bridging for very big hard-core solute particles that induce thick adsorbed (wetting) films, but some new features arise for soft-core systems. At first sight we might expect the first (single particle insertion) term in Eq. (16) to be inaccurate as $h \rightarrow 0$, when the big particles are strongly overlapping. However, this is not the case. When $h=0$ the first term in Eq. (16) is accurate, since two big particles lying on top of each other result in an external potential that has the same form as that due to a single big particle with $\epsilon_{B \nu}$ twice the value for one of the big particles taken alone. In other words, if we take the first term in Eq. (15) and make the substitution $\epsilon_{B \nu} \rightarrow 2 \epsilon_{B \nu}$, then we obtain the same first term as in Eq. (16). Given this observation the first term in Eq. (16) should be accurate for both large $h$ and for $h=0$. Thus, by continuity we expect it to be accurate for all values of $h$. The overall accuracy of Eq. (16) should depend upon how accurately we determine the surface area $A(l, h)$ which appears in the second term.

Using Eqs. (16), (15), and (3) we can obtain an expression for the SM potential,

$$
W_{B B}(h) \simeq\left[A(l, h)-8 \pi l^{2}\right] \gamma(\infty) .
$$

We now present a simple model for $A(l, h)$ (see also Ref. 32), which we expect to be reliable for values of $h$ near to where the bridging transition occurs.
When $h \gg 2 l$, i.e., no fluid bridge is present, then $A(l, h)=8 \pi l^{2}$, and Eq. (17) gives $W_{B B}(h)=0$. When there is a bridge, we approximate the end sections of $A(l, h)$ by the surfaces of two sections of spheres with radius $l$, and the bridge surface by the surface generated by rotating the arc of a circle, of radius $s$, about the axis passing through the centers of the end sphere sections (the $z$ axis). We denote the width along the $z$ axis of the bridge section by $2 w$ and the diameter of the bridge section at the midpoint between the centers of the end sphere sections by $2 d$. The surface area of the two end spherical sections is $4 \pi l(l+h / 2-w)$ and the surface area of the bridge section is $4 \pi s(s+d) \arcsin (w / s)$ $-4 \pi s w$. Requiring continuity of the surfaces where the end and bridge sections meet and also requiring continuity in the gradients at the point where these sections join, we eliminate $s$ and $d$ to obtain the following expression for the total surface area:

$$
\begin{aligned}
A(l, h)= & \frac{2 \pi w l h}{(h / 2-w)^{2}} \sqrt{l^{2}-(h / 2-w)^{2}} \arcsin \left(\frac{h / 2-w}{l}\right) \\
& -\frac{4 \pi w^{2} l}{h / 2-w}+4 \pi l(l+h / 2-w) .
\end{aligned}
$$

We choose the value $w=w_{0}$ which minimizes $A$, i.e., $\partial A /\left.\partial w\right|_{w=w_{0}}=0$ and use this prescription for calculating $A(l, h)$ with Eq. (17) to calculate the SM potential between two big GCM particles at state points near to coexistence. For the case when the small particle solvent has a total density $\rho^{0} R_{11}^{3}=8.5$ and concentration $x=0.948$, corresponding to the full DFT calculation of the SM potential in Fig. 6, we find that $\beta R_{11}^{2} \gamma(\infty)=0.830$, and that the film thickness $l / R_{11} \simeq 7$ (see Fig. 6 in Ref. 9). Using these values in Eqs. (18) and (17), we calculate the SM potential for this state point. The result is the dot-dashed line displayed in Fig. 6 which is in good qualitative agreement with our results from the full brute-force calculation of the SM potential, particularly for values of $h$ near to where the bridging transition occurs. We also used this simple approximation for the case when the small particle solvent has a total density $\rho^{0} R_{11}^{3}$ $=6.9$ and concentration $x=0.88$, corresponding to the full DFT calculation of the SM potential in Fig. 7. For this state point $\beta R_{11}^{2} \gamma(\infty)=0.152$ and $l / R_{11}=9.6$ (see Figs. 4 and 9 in Ref. 9), and the SM potential is shown as the dot-dashed line in Fig. 7. Again, the results are in qualitative agreement with those of the full calculation. In particular, this simple approach provides a surprisingly accurate means of estimating the value of $h$ at which the bridging transition will occur. If we assume that bridging will only occur when $W_{B B}(h)<0$ the resulting values of $h_{t}$ underestimate the results of the full calculation by only a few percent in both cases. Even for small values of $h$ the results of the sharp-kink approximation for $W_{B B}(h)$ are of the correct magnitude. However, this approximation fails to reproduce the correct shape of $W_{B B}(h)$ for small $h$. 


\section{THICK ADSORBED FILMS ON COMPOSITE PARTICLES}

In the previous sections we considered only state points near the binodal where we know that a single big GCM particle is "wet" by a thick adsorbed film of the coexisting phase rich in species 1, i.e., state points inside or below the single particle thin-thick adsorbed film transition line. However, there can also be pronounced effects on the SM potential due to the presence of thick adsorbed films for state points outside the single particle thin-thick adsorbed film transition line (see Fig. 1), where a single big particle immersed in the solvent does not develop a thick adsorbed film. When two big particles are sufficiently close together the resulting composite object can be sufficiently large that a thick film is adsorbed. This effect is somewhat analogous to the case for big hard-core solute particles, where for certain state points for which no thick adsorbed films are present, capillary condensation of the coexisting phase can occur in the space between the two big particles, provided these come sufficiently close together. ${ }^{4,22}$ We cannot strictly describe the phenomenon we observe as capillary condensation because the big particles that we consider in the present work have soft cores. Nevertheless, the present phenomenon has a similar effect on the SM potential, i.e., there is a jump in the SM force on reducing the separation $h$. As mentioned above, this phenomenon occurs outside (but close to) the single particle thin-thick adsorbed film transition line. However, its occurrence is restricted to a particular region of the phase diagram. If one considers two big particles with full overlap $(h=0)$ one can calculate the thin-thick adsorbed film transition line for this composite object. This line is higher in total density than the corresponding single particle transition line (see Fig. 1) and serves as an upper bound for the regime where capillary condensation occurs; the latter is restricted to the region between the two transition lines.

The solvent density profiles around two big particles with $h$ sufficiently small that this condensation has occurred are very similar in form to the profiles in Fig. 4, i.e., the condensation does not just occur in the space between the two big particles, as would be the case with a pair of hardcore big particles. Rather, due to the soft-core nature of the GCM, the adsorbed film spreads around the whole region in which the two big particles are situated.

In Fig. 8 we display the SM potential between two big GCM particles in a binary solvent of smaller particles with total bulk density $\rho^{0} R_{11}^{3}=11$ and concentration $x=0.983$. This state point is located at bulk coexistence above the single particle thin-thick adsorbed film transition line but inside the transition line for the composite particle-see Fig. 1. For large values of $h$ the SM potential calculated via the bruteforce approach is in good agreement with the results from the insertion method. At this state point the insertion method does not include any effects of thick adsorbed films since the inputs into this theory are the density profiles around a single big particle; for this state point a single big particle has no thick adsorbed film. However, as $h$ is decreased the results of the full DFT calculation show that there is a discontinuity in the gradient of $W_{B B}(h)$ due to the formation of a thick ad-

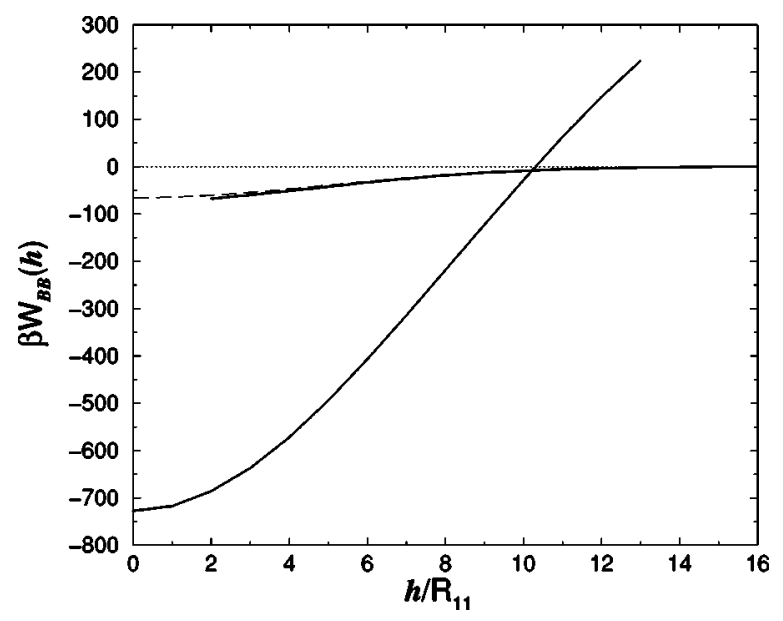

FIG. 8. The SM potential between two big GCM particles in a binary solvent of smaller particles with total bulk density $\rho^{0} R_{11}^{3}=11$ and concentration $x=0.983$ (this state point is at bulk coexistence, outside the single particle thin-thick adsorbed film transition line, but inside the thin-thick adsorbed film transition line for a composite pair of completely overlapping big particles-see Fig. 1). $h$ is the separation between the centers of the two big GCM particles. The dashed line is the result for $W_{B B}(h)$ obtained using the insertion method and the solid lines denote the results from the bruteforce method. For this state point a single big particle does not develop thick adsorbed film, but when two big particles are sufficiently close together the resulting composite object can develop a thick adsorbed film. The two branches of $W_{B B}(h)$ correspond to configurations without adsorbed films (stable at large $h$ ) and with films (stable at small $h$ ). These cross at $h / R_{11}$ $=10.2$, resulting in a discontinuity in the gradient of $W_{B B}(h)$ and a jump in the SM force.

sorbed film around the two particles. The change in the SM potential is very pronounced; the potential becomes much more strongly attractive- - see Fig. 8. The insertion method (dashed line) accounts extremely well for the large $h$ behavior of the SM potential. It also describes accurately the metastable portion of $W_{B B}(h)$ for $h$ below the transition value. However, it fails completely to describe the stable, strongly attractive portion arising from the formation of the thick adsorbed film around the two particles; it underestimates the strength of the attraction by a factor of about 10 . This is not too surprising, given that this method inputs only the density profiles around a single big particle and that these exhibit no thick adsorbed films for this state point.

\section{BRIDGING AND THE BRIDGE FUNCTION}

We recall that the SM potential $W_{B B}(r)$ is related via Eqs. (4) and (5) to the pair correlation function between solute particles, $g_{B B}(r)$, in a bulk ternary mixture which consists of a single, big solute species $B$ and two solvent species, considered in the dilute limit of solute, $\rho_{B} \rightarrow 0$. Since integral equations are a standard tool to determine bulk pair correlation functions in the theory of classical liquids, ${ }^{24}$ it is natural to analyze the SM potential within this framework. However, we recall from the outset that while integral equation theories have achieved remarkable precision in the description of one-component bulk fluids, integral equation closure approximations are generally less reliable in multicomponent mixtures, especially for situations where the size of onecomponent becomes much larger than the others leading to 
the possibility of thick film adsorption or wetting phenomena or, in the case of hard-sphere mixtures, to depletion phenomena.

Before we analyze $g_{B B}(r)$ in the ternary mixture, it is instructive to point out some features of the two-component solvent mixture which provide a relationship between the hypernetted chain (HNC) integral equations and the RPA density functional used in the present work. Diagrammatic analysis yields the following standard relationships between the pair correlation functions in a homogeneous (bulk) mixture: ${ }^{24}$

$$
\begin{aligned}
& h_{i j}(r)-c_{i j}^{(2)}(r)=\sum_{k=1,2} \rho_{k}^{0} \int \mathrm{d} \mathbf{r}^{\prime} h_{i k}\left(\left|\mathbf{r}-\mathbf{r}^{\prime}\right|\right) c_{k j}^{(2)}\left(r^{\prime}\right), \\
& \ln g_{i j}(r)+\beta v_{i j}(r)=h_{i j}(r)-c_{i j}^{(2)}(r)+b_{i j}(r),
\end{aligned}
$$

where $h_{i j}(r)=g_{i j}(r)-1$. The first equation is the OZ equation for binary mixtures and the second provides the formally exact closure to the $\mathrm{OZ}$ equation in terms of the (generally unknown) bridge function $b_{i j}(r)$. The bulk densities of the two solvent species are denoted by $\rho_{k}^{0}(k=1,2)$. It is a special feature of the binary GCM (or related soft-core models) that its pair correlation functions are very well described within the HNC approximation ${ }^{1,12-18,27,33}$ which amounts to setting $b_{i j}(r)=0$. We denote the corresponding solution for the pair direct correlation function by $c_{i j}^{(2), H N C}(r)$. The relation to density functional theory follows by noting that the HNC equations, Eq. (20) with $b_{i j}(r)=0$, are identical to the test particle equations obtained from a DFT with the excess free energy functional $^{34}$

$$
\begin{aligned}
\mathcal{F}_{e x}^{H N C}\left[\left\{\rho_{i}\right\}\right]= & A_{e x}\left(\left\{\rho_{i}^{0}\right\}\right)+\sum_{i=1,2} \int d \mathbf{r} \mu_{i}^{H N C} \Delta \rho_{i}(\mathbf{r}) \\
& -\frac{1}{2 \beta} \int d \mathbf{r} \int d \mathbf{r}^{\prime} \sum_{i j=1,2} c_{i j}^{(2), H N C}\left(\left|\mathbf{r}-\mathbf{r}^{\prime}\right|\right) \\
& \times \Delta \rho_{i}(\mathbf{r}) \Delta \rho_{j}\left(\mathbf{r}^{\prime}\right),
\end{aligned}
$$

corresponding to a Taylor expansion to quadratic order in $\Delta \rho_{i}(\mathbf{r})$ about the bulk densities. The test particle equations follow by choosing as external potentials the interparticle potential $v_{j i}(r)$, minimizing the HNC grand potential functional with respect to $\rho_{j}(r)$ and identifying $g_{j i}(r) \equiv \rho_{j}(r) / \rho_{j}^{0}$. In Eq. (21), $\Delta \rho_{i}(\mathbf{r})=\rho_{i}(\mathbf{r})-\rho_{i}^{0}$ and $A_{e x}\left(\left\{\rho_{i}^{0}\right\}\right)$ denotes the excess Helmholtz free energy of the bulk solvent. The HNC chemical potential is given by

$$
\begin{aligned}
\beta \mu_{i}^{H N C}= & \sum_{j=1,2} \rho_{j}^{0} \int d \mathbf{r}\left(\frac{1}{2} h_{j i}(r)\left[h_{j i}(r)-c_{j i}^{(2), H N C}(r)\right]\right. \\
& \left.-c_{j i}^{(2), H N C}(r)\right) .
\end{aligned}
$$

Previous results for the GCM showed that the pair correlation functions obtained from the HNC were similar to those obtained from the RPA ${ }^{1,12,13,33}$ and that the fluid-fluid binodals from the RPA and the HNC approximation were close to each other. ${ }^{33}$ If one neglects the weak density dependence of $c_{j i}^{(2), H N C}(r)$ and sets $c_{j i}^{(2), H N C}(r) \simeq c_{j i}^{(2), R P A}(r)=-\beta v_{j i}(r)$ then one can show for the binary mixture, $i=1,2$,

$$
\mathcal{F}_{e x}^{H N C}\left[\left\{\rho_{i}\right\}\right] \simeq \mathcal{F}_{e x}^{R P A}\left[\left\{\rho_{i}\right\}\right],
$$

where $\mathcal{F}_{e x}^{R P A}$ is the RPA functional defined in Eq. (11).

We have seen in earlier sections that for a fixed big Gaussian particle exerting an external potential on the solvent close to coexistence, the RPA functional accounts for the formation of a thick adsorbed film. It also accounts for complete wetting at a planar wall. ${ }^{17}$ Owing to the weak density dependence of $c_{i j}^{(2), H N C}(r)$ we also expect the HNC functional to describe thick film formation and complete wetting. [This is in sharp contrast to simple fluids of the LennardJones type where the harshly repulsive core in the interatomic potential induces a strong density dependence of the direct correlation function $c^{(2)}(r)$ and the HNC functional (21) fails to account for complete wetting. ${ }^{34,35}$ ]

Explicit minimization of the HNC functional for a binary mixture in the presence of an external potential due to a single solute particle yields the HNC solute-solvent integral equations. These can also be derived from the test particle equations of the HNC functional for the ternary mixture of binary solvent plus solute in the dilute limit of the solute, $\rho_{B} \rightarrow 0$. This functional is linear in $\rho_{B}(\mathbf{r})$ and is, at most, quadratic in the other density profiles. It is given by

$$
\begin{aligned}
\mathcal{F}_{\text {ex,tern }}^{H N C}= & \mathcal{F}_{\text {ex }}^{H N C}+\mu_{B}^{H N C} \int d \mathbf{r} \rho_{B}(\mathbf{r}) \\
& -\frac{1}{2 \beta} \int d \mathbf{r} \int d \mathbf{r}^{\prime} \sum_{i=1,2} c_{i B}^{(2), H N C}\left(\left|\mathbf{r}-\mathbf{r}^{\prime}\right|\right) \\
& \times \Delta \rho_{i}(\mathbf{r}) \rho_{B}\left(\mathbf{r}^{\prime}\right) .
\end{aligned}
$$

Here, $\mu_{B}^{H N C}\left(\left\{\rho_{i}^{0}\right\}\right)$ is the HNC insertion free energy (chemical potential) for inserting a single solute particle into the bulk solvent with densities $\rho_{i}^{0}(i=1,2)$. Analogously to Eq. (22), $\mu_{B}^{H N C}$ is given by

$$
\begin{aligned}
\beta \mu_{B}^{H N C}= & \sum_{i=1,2} \rho_{i}^{0} \int d \mathbf{r}\left(\frac{1}{2} h_{i B}(r)\left[h_{i B}(r)-c_{i B}^{(2), H N C}(r)\right]\right. \\
& \left.-c_{i B}^{(2), H N C}(r)\right),
\end{aligned}
$$

where the solute-solvent pair correlation function $h_{i B}(r)$ and the direct correlation function $c_{i B}^{(2), H N C}(r)$ are determined by solving the solvent-solvent and solute-solvent HNC equations. In the dilute limit of solute, the solute-solvent direct correlation function satisfies the $\mathrm{OZ}$ equation

$$
c_{i B}^{(2)}(r)=h_{i B}(r)-\sum_{j=1,2} \rho_{j}^{0} \int d \mathbf{r}^{\prime} h_{B j}\left(\left|\mathbf{r}-\mathbf{r}^{\prime}\right|\right) c_{j i}^{(2)}\left(r^{\prime}\right),
$$

for $i=1,2$. In this treatment thick adsorbed films can develop around a big solute particle and this is manifest in the density profiles of the two solvent species and thus in $h_{B j}(r)$. It follows from Eq. (26) that information about thick films is fed into $c_{i B}^{(2)}(r)$. We can deduce that whenever thick film formation occurs, $c_{i B}^{(2), H N C}(r)$ can be very different from the RPA result $-\beta v_{i B}(r)$.

We turn attention now to the solute-solute correlation functions. These are generated by employing $\mathcal{F}_{\text {ex,tern }}^{H N C}$, fixing $v_{B B}(r)$ as the external potential and minimizing the grand potential functional with respect to $\rho_{B}(r)$. One finds 


$$
\begin{aligned}
\ln g_{B B}(r)+\beta v_{B B}(r)= & \sum_{i=1,2} \rho_{i}^{0} \int d \mathbf{r}^{\prime} h_{B i}\left(\left|\mathbf{r}-\mathbf{r}^{\prime}\right|\right) \\
& \times c_{i B}^{(2), H N C}\left(r^{\prime}\right) .
\end{aligned}
$$

If one now employs the mixture $\mathrm{OZ}$ equations in the limit $\rho_{B} \rightarrow 0$ one obtains

$$
\ln g_{B B}(r)+\beta v_{B B}(r)=h_{B B}(r)-c_{B B}^{(2)}(r) .
$$

Note that the right-hand side of Eq. (27) depends on the solute-solvent correlation functions $h_{B i}(r)$ and $c_{i B}^{(2), H N C}(r)$. The former quantity is, essentially, the density profile of species $i$ around a single big particle determined by minimizing the HNC functional and the latter is given by the OZ equation (26). One might expect both quantities to be given accurately by the HNC treatment. The $g_{B B}(r)$ resulting from Eq. (27) yields, via Eqs. (4) and (5), an SM potential which we refer to as $W_{B B}^{H N C}(r)$ since this is consistent with the fact that $g_{B B}(r)$ satisfies Eq. (28), the HNC equation for big-big correlations; the latter sets the bridge function $b_{B B}(r)=0$.

As the HNC inputs only the pair direct correlation functions $c_{i j}^{(2), H N C}(r)$ of the small solvent species, which should be well described by their RPA counterparts, we adopt the following procedure: determine the density profiles of the two small species around a single big particle by minimizing the RPA grand potential functional, Eqs. (6) and (11), and use these as input for $h_{B i}(r)$, along with $c_{i j}^{(2), R P A}(r)$ for the solvent-solvent direct correlation functions, in Eq. (26). The resulting $c_{i B}^{(2)}(r)$ are then used in Eq. (27) to calculate $g_{B B}(r)$ and, hence, the SM potential-which should be very close to $W_{B B}^{H N C}(r)$. We find that $W_{B B}^{H N C}(r)$, for large $r$, is almost identical to the branch of $W_{B B}(r)$ obtained using the brute-force DFT method presented in Secs. II and III, for which there is no fluid bridge. In other words, when there is no fluid bridge, i.e., for $r \equiv h>h_{t}$, where $h_{t}=h_{t}\left(\rho_{1}^{0}, \rho_{2}^{0}\right)$ is the separation at which the bridging transition occurs, $W_{B B}^{H N C}(r) \simeq W_{B B}(r)$ and we can infer that the $\mathrm{HNC}$ approximation $b_{B B}(r) \simeq 0$ is valid. However, for $h<h_{t}$ we find $W_{B B}^{H N C}(r)$ is very different from $W_{B B}(r)$, indicating that the bridge function $b_{B B}(r)$, omitted from this analysis, must be substantial for $h<h_{t}$. Thus we have demonstrated that $b_{B B}(r)$ must play a significant role in determining the fluid structure when there is bridging.

We conclude that the ternary HNC functional, Eq. (24), describes correctly the bulk solvent-solvent correlations and captures thick film formation in the solute-solvent correlations with a vanishing solute-solvent bridge function, $b_{i B}(r)$ $=0$. For the solute-solute correlations the HNC assumption $b_{B B}(r)=0$ remains accurate when bridging is not present but this approximation fails completely when bridging is present. This means that a more sophisticated theory for the ternary functional should include terms proportional to $\rho_{B}(\mathbf{r}) \Delta \rho_{i}\left(\mathbf{r}^{\prime}\right) \Delta \rho_{j}\left(\mathbf{r}^{\prime \prime}\right)$ and higher orders. These will become important near the onset of the transition.

\section{DISCUSSION AND CONCLUSIONS}

Using brute-force DFT we have calculated the SM potential $W_{B B}(h)$ between a pair of big GCM particles in a binary solvent of smaller GCM particles. In particular, we have focused on the regime where the big particles are immersed in the binary solvent near to bulk phase separation, where thick films of the coexisting solvent phase adsorbed around the big particles influence strongly the SM potential. It is in this regime that we find bridging transitions. We show that the insertion method for calculating the SM potential used in Refs. 8 and 9, which is based on the ternary version of the RPA functional (11), is unable to incorporate the effects of bridging. This method does provide an accurate approximation for $W_{B B}(h)$ for solvent state points away from the binodal. The bridging that we find is of two types: (i) that due to the joining of thick adsorbed films around the individual big particles, described in Sec. III, and (ii) that due to local condensation around a pair of particles, described in Sec. V. Both result in a change in slope of $W_{B B}(h)$ at a separation $h=h_{t}$ and therefore a jump in the SM force at $h=h_{t}$.

Within our mean-field theory, bridging manifests itself as a sharp (first-order) transition. However, this cannot be the case in reality since the bridging transition involves a finite number of particles and therefore fluctuation effects will round the transition (see discussion in Ref. 4). We can make a crude estimate of the extent of rounding effects by arguing that fluctuations should only be relevant when $\mid W_{B B}^{\text {br. }}(h)$ $-W_{B B}^{\text {no br. }}(h) \mid \lesssim k_{B} T$, where $W_{B B}^{\text {br. }}(h)$ denotes the branch of $W_{B B}(h)$ where there is a fluid bridge and $W_{B B}^{\text {no br. }}(h)$ the branch without a fluid bridge. From this inequality we can obtain the width, $\delta h_{t}$, over which the transition at $h_{t}$ will be smeared. We find that $\delta h_{t} / h_{t} \sim 10^{-2}$ for the state points corresponding to the SM potentials displayed in Figs. 6 and 7. This measure of the rounding becomes smaller for solvent state points further removed from the bulk critical point. For bigger solute particles we also expect the extent of the rounding to become smaller. At first sight our estimate of the rounding may seem surprisingly small, bearing in mind that the size ratio between the big solute and small solvent particles is only about 7:1. However, due to the soft-core nature of the GCM fluid, the solvent density is high and the number of particles involved in the bridging transition can be large. This demonstrates one of the important differences between the soft-core GCM and more typical hard-core fluid systems. For hard-core particles one would not find thick adsorbed films of the solvent were the size ratio between the solute and solvent only 7:1. Typically, the solute must be of order 50 or more times larger than the solvent particles for wetting phenomena to become significant-see also the discussion in Ref. 9.

Our analysis in Sec. VI demonstrates that in order to incorporate bridging into a full (ternary) mixture theory, one must implement an accurate theory for the fluid bridge functions; in particular, for the solute-solvent and solute-solute bridge functions $b_{B i}(r)$ and $b_{B B}(r) . b_{B B}(r)$, the solute-solute bridge function, remains little understood but must play a crucial role when there are thick adsorbed films surrounding the big particles. That the bridge functions are required highlights the essential many-body nature of the effective interaction between the big solute particles. Hence, it is not surprising that the insertion method combined with the ternary version of the RPA functional (11) is unable to incorporate the effects of bridging on the SM potential. We reiterate that 
the insertion method is formally exact; it is its use with an approximate functional which leads to neglect of the key features of bridging. In order to obtain insight as to what is required in a theory for the full mixture Helmholtz free energy functional which incorporates the effect of bridging, we consider the exact inhomogeneous Kirkwood-Hill formula $^{9,36}$ (recall Eq. (9)),

$$
c_{B}^{(1)}(\mathbf{r})=-\sum_{\nu=1}^{2} \int_{0}^{1} d \lambda \int d \mathbf{r}^{\prime} \rho_{\nu}\left(\mathbf{r}^{\prime}\right) g_{B \nu}\left(\mathbf{r}, \mathbf{r}^{\prime} ; \lambda\right) \beta v_{B \nu}\left(\left|\mathbf{r}-\mathbf{r}^{\prime}\right|\right),
$$

for the one-body direct correlation function of the big solute particles in the limit $\rho_{B} \rightarrow 0 . v_{B \nu}(r)$ are the big-small pair potentials and the parameter $\lambda$, with $0 \leqslant \lambda \leqslant 1$, is used to "turn on" the effect of the inserted big particle via the potential $\lambda v_{B \nu}(r)$. One calculates the solvent response through the inhomogeneous big-small pair distribution function $g_{B \nu}\left(\mathbf{r}, \mathbf{r}^{\prime} ; \lambda\right)$, as $\lambda$ is increased from 0 to 1 . Combining Eq. (29) with Eq. (13) one obtains an exact expression for $W_{B B}(r)$, given by Eq. (70) of Ref. 9. Consider the case when the solvent is near coexistence at a state point below the single big particle thin-thick adsorbed film transition line. If one calculates $W_{B B}(h)$ via Eq. (29), then $g_{B \nu}\left(\mathbf{r}, \mathbf{r}^{\prime} ; \lambda=0\right)$ will correspond to the distribution arising from a fixed single big particle located at $\mathbf{r}=-\mathbf{h} / 2$ exerting an external potential on the solvent. This big particle will be surrounded by a thick adsorbed film. Then, turning on the effect of the second big particle (by increasing $\lambda$ from zero) located at $\mathbf{r}=+\mathbf{h} / 2$ one could perhaps envisage the situation where there might be two "jumps" in $g_{B \nu}\left(\mathbf{r}, \mathbf{r}^{\prime} ; \lambda\right)$ for a particular value of $h=|\mathbf{h}|$. The first would be at $\lambda=\lambda_{1}$, when the potential $\lambda_{1} v_{B \nu}(r)$ becomes sufficiently strong to induce condensation of the coexisting solvent phase around this second big particle. This jump in $g_{B \nu}\left(\mathbf{r}, \mathbf{r}^{\prime} ; \lambda\right)$ could then be followed by a second jump at $\lambda=\lambda_{2}\left(\lambda_{1}<\lambda_{2}<1\right)$, when a fluid bridge forms between the two big particles. That such complex phenomena must be described by $c_{B}^{(1)}(\mathbf{r})$, which is obtained by taking one functional derivative of the excess Helmholtz free energy functional Eq. (9), attests to the degree of sophistication required in the ternary mixture functional $\mathcal{F}_{e x}\left[\left\{\rho_{i}\right\}\right]$ if this is to incorporate bridging. By employing the RPA functional in the insertion approach one incorporates the effect of the thick film surrounding the first big particle via $\rho_{\nu}(\mathbf{r})$ in Eq. (29), but neglects the effect of the thick film around the second big particle by setting $g_{B \nu}\left(\mathbf{r}, \mathbf{r}^{\prime} ; \lambda\right)=1$ for all $\lambda .{ }^{9}$ Thereby one is unable to incorporate the effect of bridging on the SM potential. This point is highlighted further by the case described in Sec. V, where a single big particle has no thick adsorbed film and the bridging arises from condensation around a pair of big particles. In this situation all the information about bridging/wetting must be generated in $c_{B}^{(1)}(\mathbf{r})$ from a source other than the solvent density profiles around a single big particle, i.e., from subtle correlations in the inhomogeneous solvent. Incorporating such correlations is a tall order for a theory.

The simple capillarity (or sharp-kink) approximation used in Sec. IV to provide an approximate theory for when bridging occurs between two thick adsorbed films seems to be quite good. The simple form, Eq. (17), taken with Eq. (18), is surprisingly reliable in determining an approximation for $h_{t}$, the separation between the big particles at which bridging occurs, as well as providing a reasonable approximation for the slope of $W_{B B}(h)$ near the onset of the bridged configuration, i.e., it provides quite a good approximation for the SM force at $h \sim h_{t}$. The capillarity approximation is not reliable for small $h$. Here the shape of the SM potential determined from the capillarity approximation is completely wrong, and therefore the SM force obtained from this approximation will be completely unreliable-see Figs. 6 and 7. The brute-force calculation shows that as $h \rightarrow 0$, the SM force $\rightarrow 0$, whereas the capillarity approximation shows the SM force tending to a nonzero constant value as $h \rightarrow 0$. We believe the origin of this failure lies in our simple approximation (18) for the area of the fluid-fluid interface.

One issue we have not raised so far is what does one take for $v_{B B}(r)$, the bare big-big pair potential? This does not enter our calculation of the SM potential, since in the latter it is only $v_{B \nu}(r)$, the big-small pair potentials, that are involved; the big particles are treated as external potentials. Therefore, in principle, $v_{B B}(r)$ could take any form, although choosing a bare potential with a hard core would be inconsistent with the soft-core nature of $v_{B \nu}(r)$. A Gaussian potential of the form given in Eq. (1) would seem a natural choice for $v_{B B}(r)$. When one considers the GCM to be a simple model for polymers in solution, then the following empirical rules for the pair potential parameters apply between unlike species: ${ }^{9,13,16,20} R_{i \neq j}^{2}=\left(R_{i i}^{2}+R_{j j}^{2}\right) / 2$ and $\epsilon_{i \neq j}<\epsilon_{i i} \simeq \epsilon_{j j}$. Therefore, the choices $R_{B B} / R_{11}=7$ and $\epsilon_{B B}=2 k_{B} T$ would be consistent with the parameters we have used for the big-small pair potentials. ${ }^{9}$ If we employ a bare Gaussian potential with these parameters the big-big repulsion is negligible when compared to the attractive $W_{B B}(r)$, particularly when there are thick adsorbed wetting films present around the big particles. Thus, the resulting effective pair potential, $v_{B B}^{e f f}(r)$, given by Eq. (4) can be very strongly attractive.

If one were seeking to investigate experimentally the effects of thick adsorbed films and bridging between colloidal particles, one approach is to perform light scattering experiments in order to measure the second virial coefficient, $B_{2}{ }^{37}$ As pointed out in Ref. $9, B_{2}$, which measures the integral of $-r^{2}\left(\exp \left[-\beta v_{B B}^{e f f}(r)\right]-1\right)$ should be very large and negative when adsorbed films are present. A rapid change to large negative values of $B_{2}$ upon changing the solvent state point should indicate the development of thick adsorbed films around the colloids, thereby influencing the SM potential. ${ }^{9}$ Whether $B_{2}$ does show a rapid variation with composition in the neighborhood of the thin-thick transition lines remains to be ascertained.

Finally we note that since a pair of big particles with $h$ $=0$ exhibits a thin-thick adsorbed film transition line, at total densities higher than the single particle thin-thick adsorbed transition line (see Fig. 1), there should also be a thin-thick adsorbed film transition line at even higher densities for three big particles whose centers coincide. This will have implications for the three-body interactions between the big par- 
ticles. Furthermore, there may be other transition lines corresponding to four, five, or more big particles completely overlapping.

\section{ACKNOWLEDGMENTS}

The authors thank H. Stark for illuminating discussions and for providing a preprint of Ref. 22. We also benefited from conversations with D. Andrienko and O.I. Vinogradova. A.J.A. is grateful for the support of EPSRC under Grant No. GR/S28631/01. M.O. thanks the Alexander von Humboldt Foundation for making his stay in Bristol possible.

${ }^{1}$ C. N. Likos, Phys. Rep. 348, 267 (2001).

${ }^{2}$ R. Roth, R. Evans, and S. Dietrich, Phys. Rev. E 62, 5360 (2000).

${ }^{3}$ See the review by S. Dietrich in Phase Transitions and Critical Phenomena, 12th ed. edited by C. Domb and J. L. Lebowitz (Academic, London, 1988), p. 1.

${ }^{4}$ C. Bauer, T. Bieker, and S. Dietrich, Phys. Rev. E 62, 5324 (2000).

${ }^{5}$ J. N. Israelachvili, Intermolecular and Surface Forces, 2nd ed. (Academic, London, 1992).

${ }^{6}$ K. Lum, D. Chandler, and J. D. Weeks, J. Phys. Chem. B 103, 4570 (1999).

${ }^{7}$ D. Beysens and T. Narayanan, J. Stat. Phys. 95, 997 (1999), and references therein.

${ }^{8}$ A. J. Archer, R. Evans, and R. Roth, Europhys. Lett. 59, 526 (2002).

${ }^{9}$ A. J. Archer and R. Evans, J. Chem. Phys. 118, 9726 (2003).

${ }^{10}$ F. H. Stillinger, J. Chem. Phys. 65, 3968 (1976).

${ }^{11}$ F. H. Stillinger and D. K. Stillinger, Physica A 244, 358 (1997).

${ }^{12}$ A. Lang, C. N. Likos, M. Watzlawek, and H. Löwen, J. Phys.: Condens. Matter 12, 5087 (2000).

${ }^{13}$ A. A. Louis, P. G. Bolhuis, and J-P. Hansen, Phys. Rev. E 62, 7961 (2000).

${ }^{14}$ A. A. Louis, P. G. Bolhuis, J-P. Hansen, and E. J. Meijer, Phys. Rev. Lett. 85, $2522(2000)$.

${ }^{15}$ P. G. Bolhuis, A. A. Louis, J-P. Hansen, and E. J. Meijer, J. Chem. Phys. 114, 4296 (2001).

${ }^{16}$ A. J. Archer and R. Evans, Phys. Rev. E 64, 041501 (2001).

${ }^{17}$ A. J. Archer and R. Evans, J. Phys.: Condens. Matter 14, 1131 (2002).

${ }^{18}$ R. Finken, A. A. Louis, and J-P. Hansen, J. Stat. Phys. 110, 1015 (2003).

${ }^{19}$ P. J. Flory and W. Krigbaum, J. Chem. Phys. 18, 1086 (1950).

${ }^{20}$ J. Dautenhahn and C. K. Hall, Macromolecules 27, 5399 (1994).

${ }^{21}$ R. Evans, in Fundamentals of Inhomogeneous Fluids, edited by D. Hend- erson (Dekker, New York, 1992), Chap. 3.

${ }^{22}$ H. Stark, J. Fukuda, and H. Yokoyama, Phys. Rev. Lett. 92, 205502 (2004).

${ }^{23}$ D. Andrienko, P. Patrício, and O. I. Vinogradova, J. Chem. Phys. 121, 4414 (2004)

${ }^{24}$ J.-P. Hansen and I. R. McDonald, Theory of Simple Liquids, 2nd ed. (Academic, London, 1986).

${ }^{25}$ S. Amokrane and J. G. Malherbe, J. Phys.: Condens. Matter 13, 7199 (2001).

${ }^{26}$ D. Henderson and M. Plischke, J. Chem. Phys. 97, 7822 (1992).

${ }^{27}$ A. J. Archer, C. N. Likos, and R. Evans, J. Phys.: Condens. Matter 16, L297 (2004).

${ }^{28}$ H. H. von Grünberg and R. Klein, J. Chem. Phys. 110, 5421 (1999).

${ }^{29}$ F. Schlesener, A. Hanke, and S. Dietrich, J. Stat. Phys. 110, 981 (2003).

${ }^{30}$ In order to locate the single particle thin-thick adsorbed film transition line, one approach is to calculate density profiles at a series of state points along a particular path in the phase diagram. If one uses as a starting approximation in the numerical (iterative) procedure for calculating the density profiles those profiles corresponding to the previous point on the path, then one finds at some state point there is a jump from a thin to a thick adsorbed film. However, this jump does not occur at the equilibrium transition, rather it occurs at the "spinodal" for this single particle thinthick adsorbed film transition. In order to locate correctly the equilibrium transition line, one must calculate the grand potential corresponding to the profiles with a thick and those with a thin adsorbed film. The proper location for the transition is where these two grand potential curves cross. Near the thin-thick adsorbed film critical point there is little hysteresis and therefore very little difference between the location of the line of equilibrium transitions and the spinodal lines. However, for the present system well away from the critical point, there can be very substantial hysteresis. In Ref. 9 a spinodal line was calculated rather than the true transition line, plotted here in Fig. 1. Similar problems of metastability were elucidated in recent DFT studies of layering transitions for a model colloid-polymer mixture adsorbed at a hard wall (Ref. 31).

${ }^{31}$ P. P. F. Wessels, M. Schmidt, and H. Löwen, J. Phys.: Condens. Matter 16, S4169 (2004).

${ }^{32}$ J. Dzubiella and J.-P. Hansen, J. Chem. Phys. 121, 5514 (2004).

${ }^{33}$ A. Archer, C. N. Likos, and R. Evans, J. Phys.: Condens. Matter 14, 12031 (2002).

${ }^{34} \mathrm{M}$. Oettel, cond-mat/0410185.

${ }^{35}$ R. Evans, P. Tarazona, and U. Marini Bettolo Marconi, Mol. Phys. 50, 993 (1983).

${ }^{36}$ J. R. Henderson, Mol. Phys. 50, 741 (1983).

${ }^{37}$ See, for example, H. de Hek, and A. Vrij, J. Colloid Interface Sci. 88, 258 (1982). 